Geometry $\&$ Topology

Volume 8 (2004) 1361-1384

Published: 25 October 2004

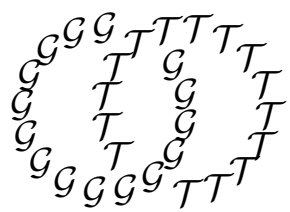

\title{
Commensurations of the Johnson kernel
}

\author{
TARA E BREndLE \\ DAN MARgalit \\ Department of Mathematics, Cornell University \\ 310 Malott Hall, Ithaca, NY 14853, USA \\ and \\ Department of Mathematics, University of Utah \\ 155 S 1440 East, Salt Lake City, UT 84112, USA \\ Email: brendle@math.cornell.edu, margalit@math.utah.edu
}

\begin{abstract}
Let $\mathcal{K}$ be the subgroup of the extended mapping class group, $\operatorname{Mod}(S)$, generated by Dehn twists about separating curves. Assuming that $S$ is a closed, orientable surface of genus at least 4 , we confirm a conjecture of Farb that $\operatorname{Comm}(\mathcal{K}) \cong \operatorname{Aut}(\mathcal{K}) \cong \operatorname{Mod}(S)$. More generally, we show that any injection of a finite index subgroup of $\mathcal{K}$ into the Torelli group $\mathcal{I}$ of $S$ is induced by a homeomorphism. In particular, this proves that $\mathcal{K}$ is co-Hopfian and is characteristic in $\mathcal{I}$. Further, we recover the result of Farb and Ivanov that any injection of a finite index subgroup of $\mathcal{I}$ into $\mathcal{I}$ is induced by a homeomorphism. Our method is to reformulate these group theoretic statements in terms of maps of curve complexes.
\end{abstract}

A correction was submitted 30 Apr 2018 and posted 27 Sep 2018 in an online supplement.

AMS Classification numbers Primary: 57S05

Secondary: 20F38, 20F36

Keywords: Torelli group, mapping class group, Dehn twist

Proposed: Walter Neumann

Seconded: Shigeyuki Morita, Joan Birman
Received: 15 June 2004

Revised: 25 October 2004 


\section{Introduction}

The extended mapping class group of a surface $S$ is:

$$
\operatorname{Mod}(S)=\pi_{0}\left(\operatorname{Homeo}^{ \pm}(S)\right)
$$

In this paper we show that certain topologically defined subgroups of $\operatorname{Mod}(S)$ have algebraic structures which strongly reflect their topological origins. We assume throughout that $S$ is a closed, oriented surface. Also, unless specifically stated otherwise, the genus $g$ of $S$ is assumed to be at least 4 .

The Torelli group $\mathcal{I}=\mathcal{I}(S)$ is the subgroup of $\operatorname{Mod}(S)$ consisting of elements which act trivially on $H=H_{1}(S, \mathbb{Z})$, and $\mathcal{K}=\mathcal{K}(S)$ denotes the subgroup of $\mathcal{I}$ generated by Dehn twists about separating curves. Birman and Powell initially suggested that $\mathcal{K}$ has finite index in $\mathcal{I}$. However, Johnson, who first singled out $\mathcal{K}$ for study in its own right, showed (among other things) that $\mathcal{K}$ arises naturally as the kernel of a map from $\mathcal{I}$ to $\wedge^{3}(H) / H$ [18]; hence we call $\mathcal{K}$ the Johnson kernel.

Building on Johnson's work, Morita showed that every homology 3-sphere is obtained by splitting $S^{3}$ along a Heegaard surface and regluing by an element of the Johnson kernel 24]. Morita further demonstrated that the natural map from $\mathcal{K}$ to $\mathbb{Z}$, given by computing the Casson invariant of the associated homology 3 -sphere, is a homomorphism (this is not true for the analagous map from $\mathcal{I}$ to $\mathbb{Z}$ ). More recently, Biss and Farb applied ideas of McCullough and Miller [23] to prove that $\mathcal{K}$ is not finitely generated [3].

Abstract commensurators The abstract commensurator of a group $\Gamma$, denoted $\operatorname{Comm}(\Gamma)$, is the group of isomorphisms of finite index subgroups of $\Gamma$ (under composition), with two such isomorphisms equivalent if they agree on a finite index subgroup of $\Gamma$. The product of $\phi: G \rightarrow H$ with $\psi: G^{\prime} \rightarrow H^{\prime}$ is a map defined on $\phi^{-1}\left(H \cap G^{\prime}\right)$.

While there is always a map from $\operatorname{Aut}(\Gamma)$ to $\operatorname{Comm}(\Gamma)$, one generally expects $\operatorname{Comm}(\Gamma)$ to be much larger (for instance, if $\Gamma$ is arithmetic). As an example, $\operatorname{Comm}\left(\mathbb{Z}^{n}\right) \cong \mathrm{GL}_{n}(\mathbb{Q})$, whereas $\operatorname{Aut}\left(\mathbb{Z}^{n}\right) \cong \mathrm{GL}_{n}(\mathbb{Z})$. On the other hand, Ivanov used Theorem 1.10 below to prove $\operatorname{Comm}(\operatorname{Mod}(S)) \cong \operatorname{Mod}(S)$ [15], Farb and Mosher showed that any word-hyperbolic surface-by-free group has finite index in its abstract commensurator [5], and Farb and Handel proved $\operatorname{Comm}\left(\operatorname{Out}\left(F_{n}\right)\right) \cong \operatorname{Out}\left(F_{n}\right)[7]$.

We confirm a conjecture of Farb, from his talk at an AMS sectional meeting in 2002 (Question 2 of [6]): 
Main Theorem 1 For $S$ a surface of genus $g \geq 4$, we have:

$$
\operatorname{Comm}(\mathcal{K}) \cong \operatorname{Aut}(\mathcal{K}) \cong \operatorname{Mod}(S)
$$

This follows from a more general theorem:

Main Theorem 2 Let $S$ be a surface of genus $g \geq 4$. Any injection $\phi: G \rightarrow$ $\mathcal{I}$, where $G$ is a finite index subgroup of $\mathcal{K}$, is induced by an element $f$ of $\operatorname{Mod}(S)$ in the sense that $\phi(h)=f h f^{-1}$ for all $h \in G$.

Co-Hopfian property A group is co-Hopfian if each of its injective endomorphisms is an isomorphism. In general, this is more rare (and harder to prove) than the Hopfian property, which, for instance, holds for all finitely generated linear groups by work of Mal'cev (recall that a group is Hopfian if every surjective endomorphism is an isomorphism).

The co-Hopfian property has been demonstrated for lattices in semisimple Lie groups by Prasad [25, torsion-free non-elementary freely indecomposable wordhyperbolic groups by Sela [26], $\operatorname{Mod}(S)$ by Ivanov and McCarthy [16], the braid group modulo its center by Bell and Margalit [1], and finite-index subgroups of $\operatorname{Out}\left(F_{n}\right)$ by Farb and Handel [7] (the case of $\operatorname{Out}\left(F_{n}\right)$ itself follows from work of Bridson and Vogtmann [4]).

As consequences of Main Theorem 2, we establish two basic algebraic properties of $\mathcal{K}$. First, $\mathcal{K}$ is co-Hopfian. Also, $\mathcal{K}$ is characteristic in $\mathcal{I}$. The former was asked by Farb as Question 3 in his lecture [6].

Corollary 1.1 If $S$ is a surface of genus $g \geq 4$, then $\mathcal{K}$ and all of its finite index subgroups are co-Hopfian.

To obtain the corollary for a finite index subgroup $G$, note that given an injection $G \rightarrow G$, the automorphism of $\mathcal{K}$ supplied by Main Theorem 2 sends each of the (finitely many) cosets of $G$ in $\mathcal{K}$ entirely into another such coset.

Corollary 1.2 If $S$ is a surface of genus $g \geq 4$, then $\mathcal{K}$ is characteristic in $\mathcal{I}$. Finite index subgroups of $\mathcal{K}$ are characteristic in $\mathcal{I}$ up to conjugacy.

By "characteristic up to conjugacy", we mean that automorphisms of $\mathcal{I}$ preserve the conjugacy class in $\mathcal{I}$ of a finite index subgroup of $\mathcal{K}$.

Our methods, which are largely inspired by the work of Farb and Ivanov, can be used recover their main results: 
Theorem 1.3 For $S$ a surface of genus $g \geq 4$, we have:

$$
\operatorname{Comm}(\mathcal{I}) \cong \operatorname{Aut}(\mathcal{I}) \cong \operatorname{Mod}(S)
$$

Theorem 1.4 If $S$ is a surface of genus $g \geq 4$, then $\mathcal{I}$ and all of its finite index subgroups are co-Hopfian. Further, any injection $\psi: G \rightarrow \mathcal{I}$, where $G$ is a finite index subgroup of $\mathcal{I}$, is induced by an element $f$ of $\operatorname{Mod}(S)$ in the sense that $\psi(h)=f h f^{-1}$ for all $h \in G$.

We remark that Farb and Ivanov proved these for $g \geq 5$, and McCarthy and Vautaw extended the result that $\operatorname{Aut}(\mathcal{I}) \cong \operatorname{Mod}(S)$ to $g \geq 3$ [22]. We also note that in the case of the Johnson kernel itself, Corollary 1.2 also follows from Theorem 1.4, since conjugation by an element of $\operatorname{Mod}(S)$ preserves the set of Dehn twists about separating curves (see Fact 2.3 in Section 2 ).

Our basic strategy is to recast algebraic statements about $\mathcal{K}$ in terms of combinatorial topology. That is, given an injection of a finite index subgroup of $\mathcal{K}$ into $\mathcal{I}$, we construct a map of the complex of separating curves $\mathrm{C}_{s}(S)$, which has vertices corresponding to separating curves in $S$ and edges corresponding to disjointness (see Section 21). We then prove:

Theorem 1.5 For $S$ a surface of genus $g \geq 4$, we have:

$$
\operatorname{Aut}\left(\mathrm{C}_{s}(S)\right) \cong \operatorname{Mod}(S)
$$

More generally, we look at superinjective maps of $\mathrm{C}_{s}(S)$. Superinjective maps were defined and used by Irmak in order to show that, for most surfaces, any injection of a finite index subgroup of $\operatorname{Mod}(S)$ into $\operatorname{Mod}(S)$ is induced by a homeomorphism [13] [12. See Section 2 for a precise definition.

Theorem 1.6 If $S$ is a surface of genus $g \geq 4$, then every superinjective map of $\mathrm{C}_{s}(S)$ is induced by an element of $\operatorname{Mod}(S)$.

The corresponding results for Torelli groups concern the Torelli complex $\mathcal{T}(S)$, which has a vertex for each separating curve and bounding pair in $S$, and edges between vertices which can be realized disjointly in $S$ (see Section 2). This complex was originally defined by Farb and Ivanov (their Torelli geometry $\mathcal{T} G(S)$ is $\mathcal{T}(S)$ with a certain marking) [8].

We prove:

Theorem 1.7 For $S$ a surface of genus $g \geq 4$, we have:

$$
\operatorname{Aut}(\mathcal{T}(S)) \cong \operatorname{Mod}(S)
$$


Theorem 1.8 If $S$ is a surface of genus $g \geq 4$, then any superinjective map of $\mathcal{T}(S)$ is induced by an element of $\operatorname{Mod}(S)$.

The previous two theorems generalize the following theorem of Farb and Ivanov about automorphisms of $\mathcal{T} G(S)$ (here, automorphisms preserve the marking) [8]:

Theorem 1.9 For $S$ a surface of genus $g \geq 5$, we have:

$$
\operatorname{Aut}(\mathcal{T} G(S)) \cong \operatorname{Mod}(S)
$$

All of the above results concerning maps of "curve complexes" are based on the seminal theorem of Ivanov about the complex of curves $\mathrm{C}(S)$ (defined in Section 21) [15]:

Theorem 1.10 For $S$ a surface of genus $g \geq 3$, we have:

$$
\operatorname{Aut}(\mathrm{C}(S)) \cong \operatorname{Mod}(S)
$$

In lower genus cases, explored by Korkmaz and Luo [19] [20, there are exceptions to Ivanov's theorem.

Remarks Despite the deep connection between mapping class groups and arithmetic groups, none of the $\operatorname{groups} \operatorname{Mod}(S), \mathcal{I}$, and $\mathcal{K}$ are arithmetic [8] [15]. For $\mathcal{K}$, it suffices to note that arithmetic groups are finitely generated and compare with the aforementioned theorem of Biss and Farb.

Mustafa Korkmaz has pointed out another approach to computing $\operatorname{Aut}\left(\mathrm{C}_{s}(S)\right.$ ), using his theorem that $\operatorname{Aut}(\mathrm{C}(S)) \cong \operatorname{Mod}(S)$ for punctured spheres. However, since there is currently no analog of Irmak's superinjectivity result for genus zero, this method does not seem to recover our second main theorem.

Outline We start with any injection:

$$
\phi: G \hookrightarrow \mathcal{I}
$$

where $G$ is any finite index subgroup of $\mathcal{K}$. As per Main Theorem 2 , we aim to produce a mapping class $f$ which induces $\phi$.

Step 1 The injection $\phi$ induces a map $\phi_{\star}$ of the vertices of $\mathrm{C}_{s}(S)$ into $\mathcal{T}(S)$. We show that $\phi$ takes a Dehn twist to either a Dehn twist or a "bounding pair map" (a product of two Dehn twists), so we get a natural map $\phi_{\star}$ from curves to collections of curves. This follows from Proposition 3.5, which relies 
on Ivanov's algebraic characterization of "simple" mapping classes - the centers of their centralizers are infinite cyclic.

Step 2 The set map $\phi_{\star}$ extends to a simplicial map from $\mathrm{C}_{s}(S)$ to $\mathcal{T}(S)$. This follows from Lemma 3.6, which uses the fact that Dehn twists commute if and only if the corresponding curves are disjoint.

Step 3 The map $\phi_{\star}$ is really a map of $\mathrm{C}_{s}(S)$ to itself. In other words, we show in Proposition 3.8 that $\phi_{\star}$ takes separating curves to separating curves. The idea is that the rank of a maximal abelian subgroup on either side of a separating curve is even, and on either side of a bounding pair is odd. We use the assumption that $S$ is closed in Section 3 .

Step 4 The map $\phi_{\star}$ extends to a map $\widehat{\phi}_{\star}$ of $\mathrm{C}(S)$. In Sections 4.1 and 4.2 , we define an action on nonseparating curves. The key is that a nonseparating curve is characterized by the separating curves which cut off a genus 1 subsurface containing that curve. To define $\widehat{\phi}_{\star}$ on a particular nonseparating curve, it suffices to choose two such separating curves - a "sharing pair". We use the assumption on genus here.

To show that $\widehat{\phi}_{\star}$ is well-defined, ie, that the map is independent of choices of sharing pairs, we apply the chain-connectedness of a certain arc complex defined by Harer.

Step 5 The map $\widehat{\phi}_{\star}$ is a superinjective simplicial map of $\mathrm{C}(S)$ to itself. As we shall see in Section 4.3. this follows easily from the superinjectivity of $\phi_{\star}$ and the definition of $\widehat{\phi}_{\star}$. It is then a theorem of Irmak that $\widehat{\phi}_{\star}$ is induced by some $f \in \operatorname{Mod}(S)$.

Step 6 Both $\phi_{\star}$ and $\phi$ itself are induced by $f$. We accomplish this by showing that $\phi(h)$ and $f h f^{-1}$ have the same action on $\mathrm{C}_{s}(S)$ for any $h \in G$. Thus, we conclude in Section [5 that $\phi$ is really the restriction of an element of $\operatorname{Aut}(\mathcal{K})$ and $\phi_{\star}$ is really an automorphism of $\mathrm{C}_{s}(S)$. To summarize:

$$
\phi \mapsto \phi_{\star} \mapsto \widehat{\phi}_{\star} \mapsto f
$$

At this point, we have established Main Theorem 2 and Theorem [1.6, and we have:

$$
\operatorname{Aut}(\mathcal{K}) \cong \operatorname{Comm}(\mathcal{K}) \rightarrow \operatorname{Mod}(S)
$$

The natural map $\eta: \operatorname{Mod}(S) \rightarrow \operatorname{Aut}(\mathcal{K})$ is an inverse, so it is an isomorphism.

In Section 6, we explain how to get the related results about Torelli groups.

Acknowledgements The authors are indebted to Bob Bell, Mladen Bestvina, Nate Broaddus, Indira Chatterji, Benson Farb, Karen Vogtmann, and Kevin 
Wortman for reading our drafts, and for useful conversations. In particular, Benson Farb suggested that we extend our earlier results to finite index subgroups. Bob Bell was especially helpful in listening to many of our proofs. We are grateful to Mustafa Korkmaz for sharing his alternate approach to the problem, and for helpful suggestions. We are also very thankful for corrections made by Walter Neumann to an earlier manuscript. Finally, we thank Martin Kassabov for pointing out that our proof works in the added case of genus four.

Both authors would like to thank Joan Birman, Benson Farb, and Kevin Wortman for their enthusiasm and encouragement on this project. We would also like to express our gratitude to Cornell University and the University of Utah for pleasant working environments during our respective visits. Finally, we are grateful to the referee for carefully reading the paper and suggesting many improvements.

The first author is partially supported by a VIGRE postdoc under NSF grant number 9983660 to Cornell University. The second author is partially supported

by a VIGRE postdoc under NSF grant number 0091675 to the University of Utah.

\section{Background}

\subsection{Curves}

When no confusion arises, we will use curve to mean either "simple closed curve" or "isotopy class of simple closed curves".

A curve $c$ is separating if $S-c$ is not connected. The genus of a separating curve $c$ on $S$ is the minimum of the genera of the two components of $S-c$.

Bounding pairs A bounding pair, denoted $(a, b)$, is an ordered pair of disjoint, homologous nonseparating curves.

Intersection We use $\mathrm{i}(\cdot, \cdot)$ to mean geometric intersection number. If either term is a bounding pair, we use the following formula:

$$
\mathrm{i}((a, b), \cdot)=\mathrm{i}(a, \cdot)+\mathrm{i}(b, \cdot)
$$

We say that two curves and/or bounding pairs intersect if their geometric intersection is nonzero. 


\subsection{Generators}

A Dehn twist about a simple closed curve $c$, denoted $T_{c}$, is the isotopy class of a homeomorphism which is the identity outside of a regular neighborhood $N$ of $c$, and which is described on the annulus $N$ by Figure 1 .

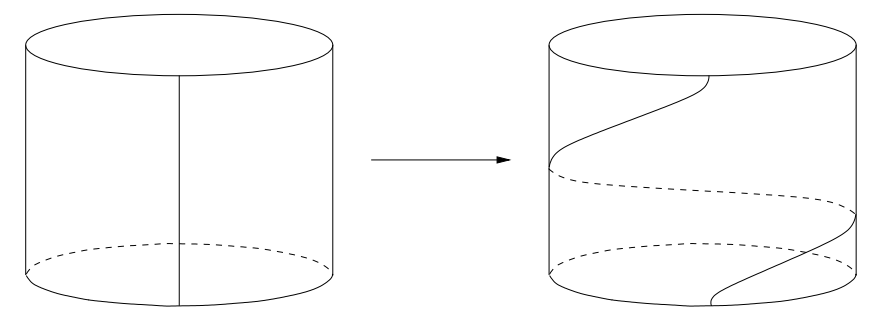

Figure 1: A Dehn twist

Bounding pair maps Johnson proved that $\mathcal{I}$ is generated by finitely many bounding pair maps [17], which are given by:

$$
B P(a, b)=T_{a} T_{b}^{-1}
$$

for $(a, b)$ a bounding pair. The Johnson kernel contains no bounding pair maps.

Rank Both Dehn twists and bounding pair maps can appear in free abelian subgroups of maximal rank in $\mathcal{I}$. In general, we denote by $\operatorname{rk}(\Gamma)$ the maximal rank of a free abelian subgroup in a group $\Gamma$. The next proposition follows from the classification of abelian subgroups of $\operatorname{Mod}(S)$ obtained by Birman, Lubotzky, and McCarthy [2].

Proposition 2.1 For any surface $S, \operatorname{rk} \mathcal{K}=\operatorname{rk} \mathcal{I}$. If $S$ has genus $g$ and $b$ boundary components where $b \in\{0,1,2\}$, then $\operatorname{rk} \mathcal{K}=\operatorname{rk} \mathcal{I}=2 g-3+b$.

In particular, Proposition 2.1tells you the size of a maximal collection of disjoint separating curves on $S$, as there is always a subgroup of rank $\operatorname{rk} \mathcal{K}=\operatorname{rk} \mathcal{I}$ generated by Dehn twists, and any collection of $n$ disjoint curves gives rise to a free abelian subgroup of rank $n$. The parities of the ranks in Proposition 2.1 are crucial in Proposition 3.8 .

\section{$2.3 \quad$ Relations}

While a complete set of relations for $\mathcal{K}$ is not known, the results of this paper only rely on the commuting relation of Fact 2.4 below. 


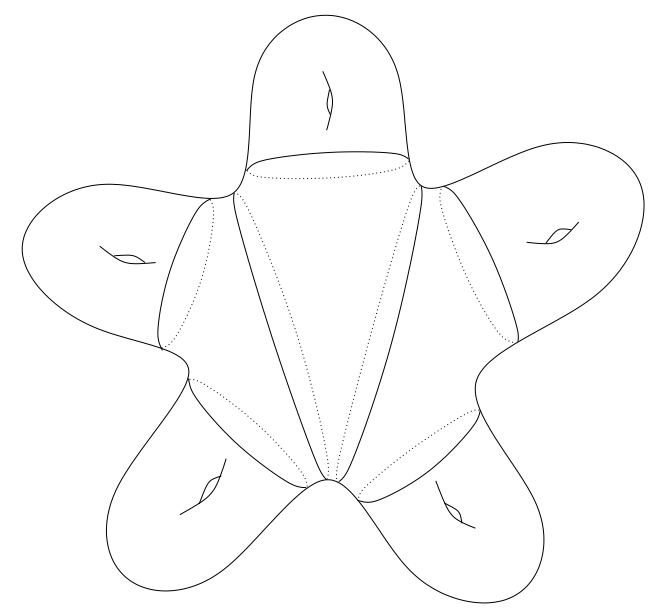

Figure 2: A maximal collection of disjoint separating curves

Fact 2.2 Let $f, T_{c} \in \mathcal{I}$, where $T_{c}$ is a Dehn twist. For $k \neq 0$, we have $\left[f, T_{c}^{k}\right]=1$ if and only if $f(c)=c$.

The last statement follows from the fact that elements of $\mathcal{I}$ are orientation preserving, together with the identity:

Fact 2.3 For $f, T_{c} \in \operatorname{Mod}(S)$, we have $f T_{c}^{k} f^{-1}=T_{f(c)}^{\epsilon k}$, where $\epsilon$ is 1 if $f$ is orientation preserving, and -1 otherwise.

As a special case of the Fact 2.2, we have:

Fact 2.4 For $f \in\left\{B P(a, b), T_{c}\right\}, h \in\left\{B P(x, y), T_{z}\right\}$, and $j$ and $k$ nonzero, we have $\left[f^{j}, h^{k}\right]=1$ if and only if the intersection number between the corresponding curves and/or bounding pairs is zero.

\subsection{Curve complexes}

We will require the use of three different abstract complexes which have vertices corresponding to curves (or bounding pairs), and have edges between vertices which can be realized disjointly.

Complex of curves The complex of curves $\mathrm{C}(S)$ for a surface $S$, defined by Harvey [10, is the abstract simplicial flag complex with a vertex for each curve in $S$ and edges between vertices which can be realized as disjoint curves in $S$. 
Complex of separating curves The subcomplex of $\mathrm{C}(S)$ spanned by vertices corresponding to separating curves is called the complex of separating curves, and is denoted $\mathrm{C}_{s}(S)$.

Farb and Ivanov introduced $\mathrm{C}_{s}(S)$ and showed that it is connected for all surfaces of genus at least 3 [8], in particular for the surfaces we consider (for alternate proofs, see [21] and [22]).

Torelli complex We denote by $\mathcal{T}(S)$ the abstract simplicial flag complex with vertices corresponding to separating curves and bounding pairs in $S$, and edges between vertices that can be realized disjointly in $S$. This Torelli complex is the simplicial complex underlying the Torelli geometry of Farb and Ivanov [8. It follows from the connectedness of $\mathrm{C}_{s}(S)$ that $\mathcal{T}(S)$ is connected.

Superinjective maps A simplicial map $\psi$ of $\mathrm{C}(S), \mathrm{C}_{s}(S)$, or $\mathcal{T}(S)$ into itself is superinjective if $\mathrm{i}(\psi(a), \psi(b)) \neq 0$ whenever $\mathrm{i}(a, b) \neq 0$. Here, we are computing intersection number on the curves (and/or bounding pairs) corresponding to the vertices of the complex.

It is not hard to prove that superinjectivity implies injectivity for all of the above complexes, but this does not follow immediately from the definition (see [13]). It does follow from the definition that superinjective maps preserve disjointness (the maps are simplicial).

Remark If $X$ is a flag complex, then $\operatorname{Aut}(X)=\operatorname{Aut}\left(X^{(1)}\right)$, where $X^{(1)}$ is the 1 -skeleton of $X$. Thus, we may restrict our attention to vertices and edges when discussing automorphisms of $\mathrm{C}(S), \mathrm{C}_{s}(S)$, and $\mathcal{T}(S)$.

\section{Separating curves}

Let $\phi: G \rightarrow \mathcal{I}$ be an injection, where $G$ is a finite index subgroup of $\mathcal{K}$. The goal of this section is to prove that the image under $\phi$ of a power of a Dehn twist in $G$ is a power of a Dehn twist. This will imply two things:

(1) $\phi(G)<\mathcal{K}$

(2) $\phi$ induces a superinjective map $\phi_{\star}$ of $\mathrm{C}_{s}(S)$.

We first show that $\phi$ takes a power of a Dehn twist to a power of either a Dehn twist or a bounding pair map. We then rule out bounding pair maps. 


\subsection{Centers of centralizers}

We now give a series of three propositions: an algebraic characterization of Dehn twists in $G$ in terms of centers of centralizers, a comparison between the centers of centralizers of an element and its image under an injection, and a characterization of Dehn twists (and bounding pair maps) in $\mathcal{I}$.

The first proposition follows from work of Ivanov which in turn rests on Thurston's theory of surface homeomorphisms [14]. First, we will require a lemma, which follows immediately from the fact that if a mapping class permutes components of a surface, it necessarily acts nontrivially on the first homology of $S$.

Lemma 3.1 If $f \in \mathcal{I}$, and $f(c)=c$ for some curve $c$, then $f$ leaves invariant the components of $S-c$.

Proposition 3.2 If $f \in G$ is a power of a Dehn twist, then $Z\left(C_{G}(f)\right) \cong \mathbb{Z}$ and $\operatorname{rk} C_{G}(f)=2 g-3$.

Proof Let $f=T_{c}^{k} \in G$ be a power of a Dehn twist. First, we know that $\operatorname{rk} C_{G}(f)=2 g-3$ by Proposition 2.1. It remains to show that $Z\left(C_{G}(f)\right) \cong \mathbb{Z}$.

Each element of $C_{G}(f)$ fixes the separating curve $c$ (Fact 2.2), and has welldefined restrictions to $S-c$ by Lemma 3.1. Suppose $h \in C_{G}(f)$ has nontrivial support on a component $S^{\prime}$ of $S-c$ (note that such a component necessarily has genus greater than one). Then there is a separating curve $a$ in $S^{\prime}$ with $h(a) \neq a$. By Fact 2.2, $h$ does not commute with nontrivial powers of $T_{a}$ (some of which are in $\left.C_{G}(f)\right)$, and hence $h \notin Z\left(C_{G}(f)\right)$. It follows that $Z\left(C_{G}(f)\right)$ can only consist of powers of $T_{c}$.

The following proposition is due to Ivanov and McCarthy [16]:

Proposition 3.3 Let $\phi: G \rightarrow G^{\prime}$ be an injection between groups with $\operatorname{rk}(G)$ $=\operatorname{rk}\left(G^{\prime}\right)<\infty$. Let $H \cong \mathbb{Z}^{\operatorname{rk}(G)}$ be a subgroup of $G$, and let $h \in H$. Then

$$
\operatorname{rk} Z\left(C_{G^{\prime}}(\phi(h))\right) \leq \operatorname{rk} Z\left(C_{G}(h)\right)
$$

We now require the following proposition of Farb and Ivanov (private communication; announced in [8]).

Proposition 3.4 An element $h$ of $\mathcal{I}$ is a power of a Dehn twist or a bounding pair map if and only if both of the following hold: 
(1) $Z\left(C_{\mathcal{I}}(h)\right) \cong \mathbb{Z}$

(2) $\operatorname{rk} C_{\mathcal{I}}(h)=2 g-3$

We now put the above results together:

Proposition 3.5 If $\phi: G \rightarrow \mathcal{I}$ is an injection for $G$ a finite index subgroup of $\mathcal{K}$, and $f=T_{c}^{k} \in G$, then $\phi(f)$ is a power of either a Dehn twist or a bounding pair map.

Proof We will show that $\phi(f)$ satisfies the two conditions of Proposition 3.4. We have that $\operatorname{rk} Z\left(C_{\mathcal{I}}(\phi(f))\right) \leq \operatorname{rk} Z\left(C_{\mathcal{K}}(f)\right)=1$, by Propositions 2.1, 3.2, and 3.3. Since the center of the centralizer of an element $h$ in a group always contains $h^{n}$ for all integers $n$, and since $\mathcal{I}$ is torsion free, we have that $\operatorname{rk} Z\left(C_{\mathcal{I}}(\phi(f))\right)$ is precisely 1 .

Since $\phi$ is an injection, we know that $\operatorname{rk} C_{\mathcal{I}}(\phi(f)) \geq \operatorname{rk} C_{\mathcal{K}}(f)=2 g-3$, with the latter equality given by Proposition 3.2. Proposition 2.1 now implies that this inequality must in fact be an equality, which proves the proposition.

By Proposition 3.5, $\phi$ gives a natural map $\phi_{\star}$ from the vertices of $\mathrm{C}_{s}(S)$ to the vertices of $\mathcal{T}(S)$ defined by:

$$
\phi\left(T_{a}^{k}\right)=T_{\phi_{\star}(a)}^{k^{\prime}} \quad \text { or } \quad \phi\left(T_{a}^{k}\right)=B P\left(\phi_{\star}(a)\right)^{k^{\prime}}
$$

In the remainder of this section, we will argue that the latter is an impossibility, and further that $\phi_{\star}$ is a superinjective map of $\mathrm{C}_{s}(S)$.

\subsection{Basic topology}

We will now show that $\phi_{\star}$ preserves some elementary topological relationships between separating curves on $S$. In particular, it will follow from Lemma 3.6 and Proposition 3.8 that $\phi_{\star}$ is a superinjective map of $\mathrm{C}_{s}(S)$.

Lemma 3.6 (Disjointness) If $a$ and $b$ are separating curves in $S$, then $\mathrm{i}(a, b) \neq 0$ if and only if $\mathrm{i}\left(\phi_{\star}(a), \phi_{\star}(b)\right) \neq 0$.

The above lemma follows immediately from Fact 2.4 and the assumption that $\phi$ is an injective homomorphism.

We define a side of a separating curve $z$ (or bounding pair $(a, b)$ ) to be a component of $S-z($ or $S-(a \cup b))$. 
Lemma 3.7 (Sides) If separating curves $a$ and $b$ are on the same side of a separating curve $z$, then $\phi_{\star}(a)$ and $\phi_{\star}(b)$ are separating curves or bounding pairs on the same side of $\phi_{\star}(z)$.

Proof If the curves $a$ and $b$ are on the same side of $z$, then there is a curve $c$ which intersects $a$ and $b$ but not $z$. Apply Lemma 3.6.

Proposition 3.8 (Genus) If $z$ is a genus $m$ separating curve, then $\phi_{\star}(z)$ is a genus $m$ separating curve. Further, if $a$ is on a genus $m$ side of $z$, then $\phi_{\star}(a)$ is on a genus $m$ side of $\phi_{\star}(z)$.

Proof First, if $z$ is a genus $m$ curve, then any maximal collection of disjoint separating curves in $S$ which contains $z$ is of the form:

$$
\left\{a_{1}, \ldots, a_{2 m-2}, z, b_{1}, \ldots, b_{2(g-m)-2}\right\}
$$

where the $a_{i}$ are on one side of $z$, and the $b_{i}$ are on the other side of $z$ (see Proposition 2.1).

By Proposition 3.5, Lemma 3.6, and Proposition 2.1, we have that the set $\left\{\phi_{\star}\left(a_{i}\right), \phi_{\star}(z), \phi_{\star}\left(b_{i}\right)\right\}$ is a maximal collection of mutually disjoint separating curves and bounding pairs. By Lemma 3.7 and Proposition 2.1, we either have that $\phi_{\star}(z)$ is a genus 1 separating curve and the $\phi_{\star}\left(a_{i}\right)$ and $\phi_{\star}\left(b_{i}\right)$ are on the same side of $\phi_{\star}(z)$, or $\phi_{\star}(z)$ is a genus $m$ separating curve and the $\phi_{\star}\left(a_{i}\right)$ and $\phi_{\star}\left(b_{i}\right)$ are on different sides of $\phi_{\star}(z)$.

If $m=1$, both cases are the same, so the image under $\phi_{\star}$ of $z$ (or any genus 1 curve) must be a genus 1 curve. Since there are always exactly $g$ genus 1 curves in any maximal collection of separating curves and bounding pairs, we can only have that $\phi_{\star}(z)$ is a genus 1 curve if $z$ was a genus 1 curve to begin with. Both statements of the proposition follow.

Note that the above proof does not work in general for surfaces with boundary.

Action on separating curves Applying Proposition 3.8 and Lemma 3.6, we now have that $\phi_{\star}$ is a superinjective map of $\mathrm{C}_{s}(S)$ defined by:

$$
\phi\left(T_{c}^{k}\right)=T_{\phi_{\star}(c)}^{k^{\prime}}
$$




\section{Nonseparating curves}

We show in this section that any superinjective map $\phi_{\star}$ of $\mathrm{C}_{s}(S)$ (in particular, the one defined in Section 3 ) can be extended to a map $\widehat{\phi}_{\star}$ of $\mathrm{C}(S)$; that is, we get a natural map on nonseparating curves as well. We will then see in Section 4.3 that $\widehat{\phi}_{\star}$ in turn gives rise to an element of $\operatorname{Mod}(S)$.

A nonseparating curve $\beta$ is uniquely determined by a pair of genus 1 separating curves with the property that $\beta$ lies on both of the corresponding genus 1 subsurfaces. This notion gives rise to the following definition.

Sharing pairs Let $a$ and $b$ be genus 1 curves bounding genus 1 subsurfaces $S_{a}$ and $S_{b}$ of $S$. We say that $a$ and $b$ share a nonseparating curve $\beta$ if $S_{a} \cap S_{b}$ is an annulus containing $\beta$ (see Figure 3) and $S-\left(S_{a} \cup S_{b}\right)$ is connected. We also say that $a$ and $b$ form a sharing pair for $\beta$.

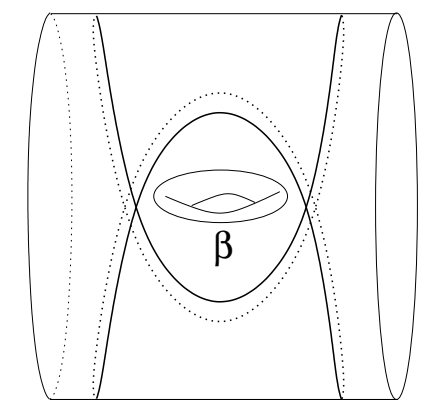

Figure 3: A sharing pair for $\beta$

Action on nonseparating curves The map $\widehat{\phi}_{\star}$ is defined on nonseparating curves as follows. If $\mathcal{P}(\beta)=\{a, b\}$ is a sharing pair for a nonseparating curve $\beta$, then $\widehat{\phi}_{\star}(\beta)$ is the curve shared by $\phi_{\star}(\mathcal{P}(\beta))$, which is defined as $\left\{\phi_{\star}(a), \phi_{\star}(b)\right\}$.

To see that $\widehat{\phi}_{\star}$ is well-defined on nonseparating curves, we must check that $\phi_{\star}(\mathcal{P}(\beta))$ is a sharing pair (Section 4.1), and that $\widehat{\phi}_{\star}(\beta)$ is independent of choice of $\mathcal{P}(\beta)$ (Section 4.2).

\subsection{Preserving sharing pairs}

We now give a characterization of sharing pairs. By the results of Section 3.2, all the properties used in the characterization are preserved by $\phi_{\star}$. The ideas here are inspired by related work of Ivanov (see [15], Lemma 1). 
Lemma 4.1 (Sharing pairs) Let $a$ and $b$ be genus 1 curves in $S$. Then $a$ and $b$ are a sharing pair if and only if there exist separating curves $w, x, y$, and $z$ in $S$ with the following properties:

- $z$ is a genus 2 curve bounding a genus 2 subsurface $S_{z}$.

- $a$ and $b$ are in $S_{z}$ and intersect each other.

- $x$ and $y$ are disjoint.

- $w$ intersects $z$, but not $a$ and not $b$.

- $x$ intersects $a$ and $z$, but not $b$.

- $y$ intersects $b$ and $z$, but not $a$.

Proof One direction is proven by construction: if $a$ and $b$ are a sharing pair, then the other curves can be chosen as in Figure 4.

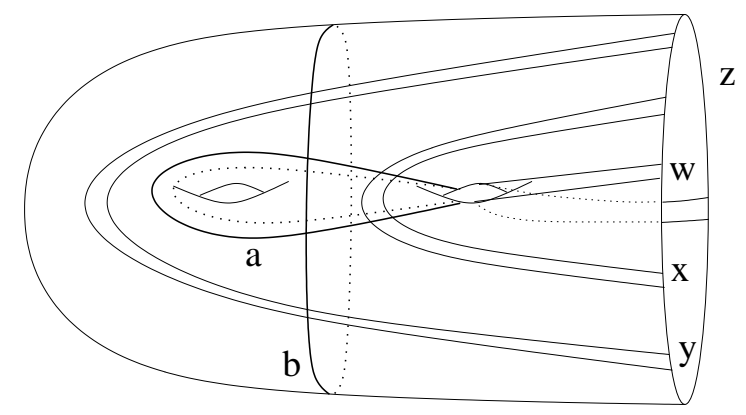

Figure 4: Characterizing curves for a sharing pair

For the other direction, we restrict our attention to $S_{z}$. On this subsurface, $a$ and $b$ are genus 1 separating curves, and each of $w, x$, and $y$ is a collection of arcs which separates $S_{z}$. Let $S_{a z}$ denote the genus 1 surface of $S_{z}$ bounded by $a$ and $z$.

Step 1 The arcs of $w$ are all parallel and nonseparating.

On $S_{a z}$, we think of $b$ as a collection of disjoint arcs with both endpoints on $a$, and of $w$ as a collection of disjoint arcs with both endpoints on $z$. By the hypotheses, the arcs of $b$ do not intersect the arcs of $w$.

Now, these arcs of $b$ and $w$ are nontrivial in $H_{1}\left(S_{a z}, a\right)$ and $H_{1}\left(S_{a z}, z\right)$, respectively: if any arcs of $w$, say, are all parallel to $z$, then $w$ and $z$ are not in minimal position; if they enclose a disc with one hole (namely, the hole is $a$ ), then there is no room for the arcs of $b$. 
If $w$ consists of non-parallel arcs, then $S_{a z}-w$ is a disk with a hole, so there is no room for the arcs of $b$.

Step $2 \quad a$ and $b$ form a sharing pair.

Let $S_{w z}$ be the genus 1 subsurface of $S_{z}$ in the complement of $w$. Each of the two boundary components of $S_{w z}$ is made up of exactly one arc from $z$ and one arc from $w$. Since $a$ and $b$ are genus 1 curves, they each split $S_{w z}$ into a torus with a hole and a disk with two holes (the boundary components of $S_{w z}$ ).

As above, at least some arcs of $x$ and $y$ must be nontrivial in the relative homology of $S_{w z}$, for otherwise the curves are not in minimal position, or they are all parallel to the arcs of $w$, which contradicts the fact that there are curves ( $a$ and $b$ ) which intersect $x$ and $y$, but not $w$. We can thus ignore the arcs of $x$ and $y$ which are parallel to $w$.

We will presently use the fact that all genus 1 curves on $S_{w z}$ (in particular, $a$ and $b$ ) are obtained by taking the boundary of a regular neighborhood in $S_{w z}$ of the union of $z \cup w$ with a defining arc connecting the boundary components of $S_{w z}$.

If the defining arcs for $a$ and $b$ intersect, then there are no choices for collections of $\operatorname{arcs} x$ and $y$ which do not intersect each other. Therefore, the defining arcs for $a$ and $b$ do not intersect (there is only one way to achieve this, topologically), and it follows that $a$ and $b$ are a sharing pair.

We remark that the collection of curves in the lemma requires genus at least 4 , hence the hypothesis in our theorems.

Lemma 4.1 implies:

Proposition 4.2 Suppose $\phi_{\star}$ is a superinjective map of $\mathrm{C}_{s}(S)$. If two genus 1 curves $a$ and $b$ in $S$ form a sharing pair, then so do $\phi_{\star}(a)$ and $\phi_{\star}(b)$.

Proof Since $a$ and $b$ share a curve, there are characterizing curves $w, x$, $y$, and $z$ as in Lemma 4.1. Since each property of this collection of curves (disjointness, sides, genus) is preserved (Lemmas 3.6 3.7 and Proposition 3.8), Lemma 4.1 implies that $\phi_{\star}(a)$ and $\phi_{\star}(b)$ share a curve. 


\subsection{Well-definedness}

We now have a map from the set of superinjective maps of $\mathrm{C}_{s}(S)$ to the set of maps of $\mathrm{C}(S)$, given by $\phi_{\star} \mapsto \widehat{\phi}_{\star}$. In order to show that this map is welldefined with respect to choice of sharing pairs, it will be more convenient for us to consider sharing pairs indirectly, via their "spines".

Spines Given two nonseparating curves $\alpha$ and $\beta$ with $\mathrm{i}(\alpha, \beta)=1$, we define $\mathcal{B}(\alpha, \beta)$ to be the genus 1 separating curve which is the boundary of a regular neighborhood of $\alpha \cup \beta$. An ordered collection of three distinct curves $\{\alpha, \beta, \gamma\}$ forms a spine for a sharing pair $\{a, b\}$ if:

$$
\begin{gathered}
\mathrm{i}(\alpha, \beta)=\mathrm{i}(\beta, \gamma)=1 \\
\mathrm{i}(\alpha, \gamma) \leq 1 \\
\mathcal{B}(\alpha, \beta)=a \quad \mathcal{B}(\beta, \gamma)=b \\
S-(\alpha \cup \beta \cup \gamma) \text { is connected }
\end{gathered}
$$

We denote this spine by $\alpha-\beta-\gamma$.

We can always choose a spine for a given sharing pair $\{a, b\}$, although this choice is not unique. If $\alpha-\beta-\gamma$ is a spine for a sharing pair with $\mathrm{i}(\alpha, \gamma)=0$, then $\alpha-\beta-T_{\beta}(\gamma)$ and $\alpha-\beta-T_{\beta}^{-1}(\gamma)$ are also spines for $\{a, b\}$. We observe that every other spine for $\{a, b\}$ is obtained by applying powers of $T_{\beta}$ (curve by curve) to these 3 spines. However, we will not use this fact.

Moves We define a move between spines to be a change of the form:

$$
\alpha-\beta-\gamma \mapsto \alpha-\beta-\gamma^{\prime}
$$

where $\gamma-\beta-\gamma^{\prime}$ is also a spine. We remark that only one curve in the corresponding sharing pairs is changed. Note that the curves $\mathcal{B}(\alpha, \beta), \mathcal{B}(\beta, \gamma)$, and $\mathcal{B}\left(\beta, \gamma^{\prime}\right)$ form three sharing pairs for $\beta$, since $\alpha-\beta-\gamma, \alpha-\beta-\gamma^{\prime}$ and $\gamma-\beta-\gamma^{\prime}$ are all spines.

A move is characterized by how many of $\mathrm{i}(\alpha, \gamma), \mathrm{i}\left(\alpha, \gamma^{\prime}\right)$, and $\mathrm{i}\left(\gamma, \gamma^{\prime}\right)$ are 1 , and how many are 0 . Using the nonseparating property of spines, it is straightforward to prove:

Lemma 4.3 Any move is topologically equivalent to one of the 4 moves shown in Figure 5 . 

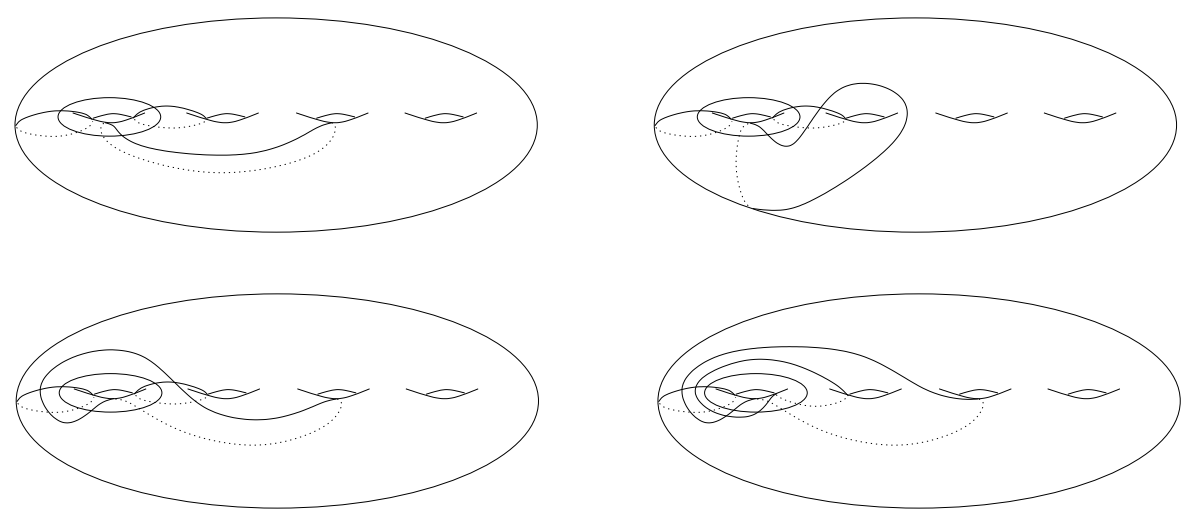

Figure 5: Moves on spines

The proof of the following lemma borrows from work of Ivanov and McCarthy (Lemma 10.11 of [16]). We will make repeated use of the following fact: if $a$ is a genus 1 separating curve, and $\alpha$ and $\beta$ are any two distinct curves on the genus 1 surface bounded by $a$, then a curve $z$ intersects $a$ if and only if it intersects at least one of $\alpha$ and $\beta$.

Lemma 4.4 Let $\beta$ be a nonseparating curve in $S$. If $\{a, b\}$ and $\left\{a, b^{\prime}\right\}$ are sharing pairs for $\beta$ which have spines differing by a move, then $\left\{\phi_{\star}(a), \phi_{\star}(b)\right\}$ and $\left\{\phi_{\star}(a), \phi_{\star}\left(b^{\prime}\right)\right\}$ are sharing pairs for the same curve.

Proof Suppose that $\alpha-\beta-\gamma$ and $\alpha-\beta-\gamma^{\prime}$ differ by a move, where $a=\mathcal{B}(\alpha, \beta)$, $b=\mathcal{B}(\beta, \gamma)$, and $b^{\prime}=\mathcal{B}\left(\beta, \gamma^{\prime}\right)$. Since $a, b$, and $b^{\prime}$ pairwise share a common curve, Proposition 4.2 implies that $\phi_{\star}(a), \phi_{\star}(b)$, and $\phi_{\star}\left(b^{\prime}\right)$ are pairwise sharing.

One can always find a separating curve $z$ which intersects $\gamma^{\prime}$ but not any of $\alpha$, $\beta$, or $\gamma$ (by Lemma 4.3). It follows that $z$ intersects $b^{\prime}$ but not $a$ or $b$, and then by Lemma 3.6, we have that $\phi_{\star}(z)$ intersects $\phi_{\star}\left(b^{\prime}\right)$ but not $\phi_{\star}(a)$ or $\phi_{\star}(b)$.

Suppose that $\phi_{\star}(a), \phi_{\star}(b)$, and $\phi_{\star}\left(b^{\prime}\right)$ do not all share the same nonseparating curve. Let $\pi-\sigma-\tau$ be a spine for $\left\{\phi_{\star}(a), \phi_{\star}(b)\right\}$. By the assumption, $\phi_{\star}\left(b^{\prime}\right)$ does not share $\sigma$ with $\phi_{\star}(a)$ and $\phi_{\star}(b)$, and hence it shares curves $\omega$ and $\nu$ with $\phi_{\star}(a)$ and $\phi_{\star}(b)$, respectively. Note that $\sigma, \omega$, and $\nu$ must all be distinct, because otherwise it follows that $\phi_{\star}\left(b^{\prime}\right)$ is equal to either $\phi_{\star}(a)$ or $\phi_{\star}(b)$.

We will now argue that there is no curve which intersects $\phi_{\star}\left(b^{\prime}\right)$ and is disjoint from both $\phi_{\star}(a)$ and $\phi_{\star}(b)$. This will contradict our earlier statement about $\phi_{\star}(z)$. Indeed, any curve $c$ which intersects $\phi_{\star}\left(b^{\prime}\right)$ must also intersect at least 
one of $\omega$ or $\nu$, say $\omega$. Since $\omega$ lies on the genus 1 subsurface bounded by $\phi_{\star}(a)$, it follows that $c$ also intersects $\phi_{\star}(a)$.

By Lemma 4.4, well-definedness of $\widehat{\phi}_{\star}$ is reduced to the following proposition, which we will now deduce from general work of Harer [9].

Proposition 4.5 Any two spines $\alpha-\beta-\gamma$ and $\delta-\beta-\epsilon$ differ by a finite sequence of moves.

Harer's complex Let $F$ be a surface with boundary, let $P$ be a finite set of points in $\partial F$, and let $P_{0}$ be a subset of $P$. Harer defines an abstract simplicial complex $X=X\left(F, P, P_{0}\right)$ with:

Vertices Isotopy classes of arcs in $F$ connecting points of $P_{0}$ to points of $P-P_{0}$.

Edges Two vertices are connected with an edge if the corresponding arcs are disjoint (apart from endpoints) and their union does not separate $F$.

In general, a $k$-simplex of $X$ is the span of $k+1$ pairwise connected edges with the property that the union of the corresponding arcs does not separate $F$.

We remark that Harer's original notation for this complex is $B X$. Let $r^{\prime}$ denote the number of boundary components of $F$ which contain a point of $P$. Harer proves:

Theorem 4.6 $X$ is spherical of dimension $2 g-2+r^{\prime}$.

By an argument of Hatcher [11, we also have:

Proposition 4.7 $X$ is chain-connected. That is, any two maximal simplices are connected by a finite sequence of maximal simplices, where consecutive simplices in the sequence share a simplex of codimension 1.

The key fact for this proposition is that the link of every simplex of codimension greater than 1 is connected, as each such link is again the complex $X$ for a surface with $2 g-2+r^{\prime}>0$ (obtained by cutting along the arcs represented by the simplex). Thus, given any path between maximal simplices, one can push the path off all simplices of codimension greater than 1 . The new path gives the sequence of maximal simplices in the statement of the proposition.

We now apply Harer's ideas to the current situation. For $\beta$ a nonseparating curve, let $F$ be the surface obtained by cutting $S$ along $\beta$. Let $P=\{p, q\}$ 
be a pair of points, one on each boundary component of $F$, and let $P_{0}=\{p\}$. Vertices of the complex $X=X\left(F, P, P_{0}\right)$ correspond to curves which intersect $\beta$ once. Further, every edge of $X$ is a spine of a sharing pair for $\beta$ (a pair of vertices fails to give a sharing pair only when the corresponding arcs lie in a 3 -holed sphere containing $\partial F$; but in this case, the arcs necessarily separate $F$, so the vertices do not form an edge). Thus, a move between spines is achieved by changing an edge to a new edge which lies in a triangle with the old edge.

Proof of Proposition 4.5 Let $\alpha-\beta-\gamma$ and $\delta-\beta-\epsilon$ be two spines for the nonseparating curve $\beta$. We think of these spines as edges in the complex $X=$ $X\left(F, P, P_{0}\right)$ defined above. Let $M$ and $N$ be any maximal simplices which contain these edges. By Proposition 4.7, there is a sequence of maximal simplices of $X$ :

$$
M=M_{0}, M_{1}, \ldots, M_{k}=N
$$

where $M_{i}$ and $M_{i+1}$ share a codimension 1 face. Using this sequence, we will construct the desired sequence of moves. Let $e_{0}$ be the edge corresponding to $\alpha-\beta-\gamma$. Now, assuming $e_{i}$ is an edge in $M_{i}$ connecting $v$ to $w$, inductively define $e_{i+1}$ as follows:

If both $v$ and $w$ are vertices of $M_{i+1}$, then $e_{i+1}$ is defined to be $e_{i}$.

In the case that $w$, say, is not a vertex of $M_{i+1}$ (note then that $v$ must be in $M_{i+1}$ ), we define $e_{i+1}$ to be the span of $v$ with any other vertex $w^{\prime}$ of $M_{i+1} \cap M_{i}$. Since $v, w$, and $w^{\prime}$ all lie in $M_{i}$, they form a triangle, and hence the edges $v w$ and $v w^{\prime}$ differ by a move.

Finally, $e_{k}$ differs from the edge corresponding to $\delta-\beta-\epsilon$ by at most two moves, since they both lie in the simplex $N$.

\subsection{Homeomorphism}

At this point, we have shown that any superinjective map $\phi_{\star}$ of $\mathrm{C}_{s}(S)$ naturally gives rise to a map $\widehat{\phi}_{\star}$ of $\mathrm{C}(S)$. In order to show that $\widehat{\phi}_{\star}$ is induced by a homeomorphism, we now check that $\widehat{\phi}_{\star}$ is a superinjective map of $\mathrm{C}(S)$. That is, for any curves $c$ and $d$, we show $\mathrm{i}(c, d) \neq 0$ if and only if $\mathrm{i}\left(\widehat{\phi}_{\star}(c), \widehat{\phi}_{\star}(d)\right) \neq 0$ :

(1) If $c$ and $d$ are both separating, then apply the fact that $\left.\widehat{\phi}_{\star}\right|_{\mathrm{C}_{s}(S)}=\phi_{\star}$ is superinjective (Lemma 3.6).

(2) If $c$ and $d$ are both nonseparating, then it suffices to note that $c$ and $d$ are disjoint if and only if there are disjoint sharing pairs representing those curves. 
(3) If $c$ is separating, and $d$ is nonseparating, then $c$ and $d$ are disjoint if and only if either $c$ is part of a sharing pair for $d$ (if $d$ is on a genus 1 side of $c$ ) or $d$ has a sharing pair whose curves are disjoint from $c$.

We appeal to the following theorem of Irmak [13]:

Theorem 4.8 For $S$ a surface of genus $g \geq 3$, any superinjective map of $\mathrm{C}(S)$ is induced by an element of $\operatorname{Mod}(S)$.

Thus, we have that $\widehat{\phi}_{\star}$ is induced by $f \in \operatorname{Mod}(S)$, which simply means that both give the same action on $\mathrm{C}(S)$.

\section{Proofs of main theorems}

In Section 3, we showed that given any injection $\phi: G \rightarrow \mathcal{K}$ for $G$ a finite index subgroup of $\mathcal{K}$, there is an associated superinjective map $\phi_{\star}$ of $\mathrm{C}_{s}(S)$. Then, in Section 4, we proved that any superinjective map $\phi_{\star}$ of $\mathrm{C}_{s}(S)$ gives rise to a superinjective map $\widehat{\phi}_{\star}$ of $\mathrm{C}(S)$, which is itself induced by a mapping class $f$. Thus, we have:

$$
\phi \mapsto \phi_{\star} \mapsto \widehat{\phi}_{\star} \mapsto f
$$

It is immediate that $\phi_{\star}$ is induced by $f$, and hence we have proven Theorem 1.6. As for the original map $\phi$, we now have that any power of a twist $T_{c}^{k} \in G$ satisfies:

$$
\phi\left(T_{c}^{k}\right)=T_{f(c)}^{k^{\prime}}
$$

for some $k^{\prime} \in \mathbb{Z}$, where $f$ is the element of $\operatorname{Mod}(S)$ given by Theorem 4.8

We now show that $f$ induces $\phi$, by which we mean that for any $h \in G$, its image is given by:

$$
\phi(h)=f h f^{-1}
$$

From this, Theorems 1.5 and 1.6, as well as Main Theorems 1 and 2, will all follow.

Let $h \in G$. Since two mapping classes which agree on all separating curves are equal, it suffices to show that for any separating curve $c$, we have $f h(c)=$ $\phi(h) f(c)$. Choose $k$ so that $T_{c}^{k}, T_{h(c)}^{k} \in G$. We have:

$$
\begin{aligned}
T_{f \circ h(c)}^{k^{\prime}} & =\phi\left(T_{h(c)}^{k}\right)=\phi\left(h T_{c}^{k} h^{-1}\right)=\phi(h) \phi\left(T_{c}^{k}\right) \phi(h)^{-1}=\phi(h) T_{f(c)}^{k^{\prime \prime}} \phi(h)^{-1} \\
& =T_{(\phi(h) \circ f)(c)}^{k^{\prime \prime}}
\end{aligned}
$$


But since Dehn twists can only be equal if they are twists about the same curve, we have:

$$
(f \circ h)(c)=(\phi(h) \circ f)(c)
$$

So it is true that $f h=\phi(h) f$, and hence $\phi(h)=f h f^{-1}$.

We have thus proven Main Theorem 2, which immediately implies that $\phi$ is the restriction to $G$ of an element of $\operatorname{Aut}(\mathcal{K})$. In particular:

$$
\operatorname{Comm}(\mathcal{K}) \cong \operatorname{Aut}(\mathcal{K})
$$

Main Theorem 1 now follows. Indeed, the natural map $\eta: \operatorname{Mod}(S) \rightarrow \operatorname{Aut}(\mathcal{K})$ is readily seen to be a right inverse of the composition of maps taking $\phi$ to $f$, and thus $\operatorname{Mod}(S) \cong \operatorname{Aut}(\mathcal{K})$. Theorem 1.5 follows similarly.

\section{Torelli group}

We will now sketch how our theorems give alternate proofs of Farb and Ivanov's results about Torelli groups.

Let $\psi: G \rightarrow \mathcal{I}$ be an injection, where $G$ is any finite index subgroup of $\mathcal{I}$. As in Farb and Ivanov's paper, $\psi$ induces a superinjective map $\psi_{\star}$ of $\mathcal{T}(S)$ (this follows from Proposition 3.4). In particular, $\psi_{\star}$ induces a superinjective map $\phi_{\star}$ of $\mathrm{C}_{s}(S)$ (by the proof of Lemma 3.8), and, following our paper, this gives rise to a mapping class $f$ which induces $\phi_{\star}$. In summary:

$$
\psi \mapsto \psi_{\star} \mapsto \phi_{\star} \mapsto f
$$

We now claim that $f$ also induces $\psi_{\star}$. It suffices to check $(f(a), f(b))=$ $\psi_{\star}((a, b))$ for any bounding pair $(a, b)$. Given $(a, b)$ choose four separating curves $w, x, y$, and $z$ as in Figure 6 .

We have that $(f(a), f(b))$ is the unique bounding pair between $f(y)$ and $f(z)$ and disjoint from $f(w)$ and $f(x)$.

Now $\psi_{\star}$ has the same action as $f$ on $w, x, y$, and $z$ since all the curves are separating. As in Section [3.2, $\psi_{\star}((a, b))$ must lie in between $f(y)$ and $f(z)$, and it must be disjoint from $f(w)$ and $f(x)$. This implies that $\psi_{\star}((a, b))=$ $(f(a), f(b))$.

By the same argument as in Section 5, $f$ induces $\psi$. Theorems 1.7 and 1.8 now follow, and from these it is straightforward to prove Theorems 1.3 and 1.4 . 


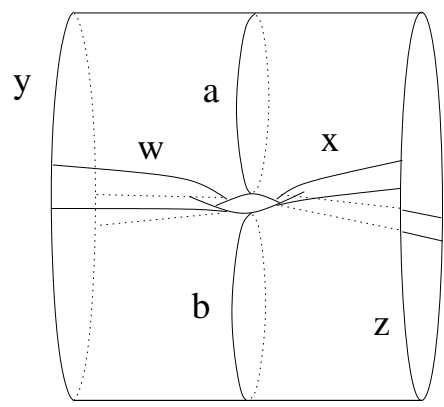

Figure 6: Separating curves defining a bounding pair

\section{References}

[1] Robert W Bell, Dan Margalit, Braid groups are almost co-Hopfian, arXiv:math.GT/0403145

[2] Joan S Birman, Alex Lubotzky, John McCarthy, Abelian and solvable subgroups of the mapping class groups, Duke Math. J. 50 (1983) 1107-1120 MathReview

[3] Daniel Biss, Benson Farb, $K_{g}$ is not finitely generated, arXiv:math.GT/0405386

[4] Martin R Bridson, Karen Vogtmann, Automorphisms of automorphism groups of free groups, J. Algebra 229 (2000) 785-792 MathReview

[5] Benson Farb, Lee Mosher, The geometry of surface-by-free groups, Geom. Funct. Anal. 12 (2002) 915-963 MathReview

[6] Benson Farb, Automorphisms of the Torelli group, AMS sectional meeting, Ann Arbor, Michigan, March 1, 2002

[7] Benson Farb, Michael Handel, Commensurations of Out $\left(F_{n}\right)$. Preprint, personal communication, September 2004

[8] Benson Farb, Nikolai V Ivanov, The Torelli geometry and its applications, arXiv:math.GT/0311123

[9] John L Harer, Stability of the homology of the mapping class groups of orientable surfaces, Ann. of Math. 121 (1985) 215-249 MathReview

[10] W J Harvey, Boundary structure of the modular group, from: "Riemann surfaces and related topics (Stony Brook, 1978)", Ann. of Math. Stud. 97, Princeton Univ. Press, Princeton, N.J. (1981) 245-251 MathReview

[11] Allen Hatcher, On triangulations of surfaces, Topology Appl. 40 (1991) 189194 MathReview

[12] Elmas Irmak, Superinjective Simplicial Maps of Complexes of Curves and Injective Homomorphisms of Subgroups of Mapping Class Groups II, arXiv:math.GT/0311407 
[13] Elmas Irmak, Superinjective simplicial maps of complexes of curves and injective homomorphisms of subgroups of mapping class groups, Topology 43 (2004) 513-541 MathReview

[14] Nikolai V Ivanov, Automorphisms of Teichmüller modular groups, from: "Topology and geometry-Rohlin Seminar", Lecture Notes in Math. 1346, Springer, Berlin (1988) 199-270 MathReview

[15] Nikolai V Ivanov, Automorphism of complexes of curves and of Teichmüller spaces, Internat. Math. Res. Notices (1997) 651-666 MathReview

[16] Nikolai V Ivanov, John D McCarthy, On injective homomorphisms between Teichmüller modular groups. I, Invent. Math. 135 (1999) 425-486 MathReview

[17] Dennis Johnson, The structure of the Torelli group. I. A finite set of generators for I, Ann. of Math. 118 (1983) 423-442 MathReview

[18] Dennis Johnson, The structure of the Torelli group. II. A characterization of the group generated by twists on bounding curves, Topology 24 (1985) 113-126 MathReview

[19] Mustafa Korkmaz, Automorphisms of complexes of curves on punctured spheres and on punctured tori, Topology Appl. 95 (1999) 85-111 MathReview

[20] Feng Luo, Automorphisms of the complex of curves, Topology 39 (2000) 283298 MathReview

[21] Howard Masur, Saul Schleimer, The Pants Complex Has Only One End, arXiv:math.GT/0312385

[22] John D McCarthy, William R Vautaw, Automorphisms of Torelli groups, arXiv:math.GT/0311250

[23] Darryl McCullough, Andy Miller, The genus 2 Torelli group is not finitely generated, Topology Appl. 22 (1986) 43-49 MathReview

[24] Shigeyuki Morita, Casson's invariant for homology 3-spheres and characteristic classes of surface bundles. I, Topology 28 (1989) 305-323 MathReview

[25] Gopal Prasad, Discrete subgroups isomorphic to lattices in semisimple Lie groups, Amer. J. Math. 98 (1976) 241-261 MathReview

[26] Z Sela, Structure and rigidity in (Gromov) hyperbolic groups and discrete groups in rank 1 Lie groups. II, Geom. Funct. Anal. 7 (1997) 561-593 MathReview 